\title{
Multiculturalismo neoliberal extractivo en la cuestión hidroeléctrica en territorio mapuche. Un análisis a la implementación de la consulta indígena en Chile'
}

\author{
Neoliberal extractive multiculturalism in the hydroelectric \\ issue in Mapuche territory. An analysis of the \\ implementation of the indigenous consultation in Chile
}

\author{
Ximena Cuadra Montoya²
}

\begin{abstract}
RESUMEN
Los conflictos por la instalación de centrales hidroeléctricas en territorio mapuche se han incrementado durante los últimos veinte años. Así también se han iniciado políticas que proponen incluir a los pueblos indígenas. En este artículo exploro cómo el multiculturalismo expresado en la implementación de la consulta indígena en proyectos hidroeléctricos en territorio mapuche se relaciona con los cambios en la política energética. Para ello procederé a exponer las líneas centrales de una definición de multiculturalismo neoliberal extractivo. Luego, daré cuenta de la cuestión hidroeléctrica en territorio mapuche, incluyendo la expansión de las energías renovables no convencionales, ERNC. Finalmente, analizo la implementación de la consulta indígena en los proyectos hidroeléctricos en el territorio mapuche, revisando especialmente las dinámicas de inclusión y exclusión que se han producido. Este artículo se basa en mi investigación doctoral en curso interesada en el análisis del lugar del Pueblo Nación Mapuche en el espacio político en el contexto de los conflictos por la hidroelectricidad. Metodológicamente, desarrollo un enfoque cualitativo de carácter inductivo que me permitió observar primero la conflictividad social y luego identificar fenómenos específicos asociados a la política del reconocimiento.

Palabras clave: multiculturalismo, consulta indígena, política energética, centrales hidroeléctricas, Pueblo Nación Mapuche
\end{abstract}

\section{ABSTRACT}

Conflicts over the installation of hydroelectric power plants in Mapuche territory have increasingly emerged over the last twenty years, and so have policies proposing to include indigenous peoples. In this article I explore how multiculturalism-as expressed in the implementation of indigenous consultation in hydroelectric projects in Mapuche territoryrelates to changes in energy policy. I first propose a definition of what I have deemed extractive neoliberal multiculturalism. I then provide an account of the hydroelectric issue

Agradezco los comentarios de las evaluaciones anónimas, así como los de Nancy Thede, Hernán Cuevas Valenzuela, Antoine Maillet, y Ange Cayumán. Esta investigación fue facilitada por el Proyecto FONDECYT No 1150788 "Nuevos Estudios De La Ciudadanía En Chile: Historia, Transformaciones Y Espacio" y el programa Bourses-Stages Banque Scotia-Institute des études internationales de Montréal (IEIM).

Estudiante de doctorado en Ciencia Política, Departamento de Ciencia Política, Université du Québec à Montréal. Correo electrónico: xcuadram@gmail.com 
in Mapuche territory, including the expansion of non-conventional renewable energies, NCRE. Finally, I analyze the implementation of indigenous consultation processes on hydroelectric projects in Mapuche territory by focusing on the dynamics of inclusion and exclusion. This article is based on my ongoing doctoral research, which seeks to analyze the place of the Mapuche Nation People in the political space in the context of conflicts over hydroelectricity. The methodology is based on an inductive qualitative approach that allows me to first observe social conflict and then identify specific phenomena associated with recognition politics

Keywords: multiculturalism, indigenous consultation, energy politics, hydroelectric dams, Mapuche Nation

\section{RESUMO}

Os conflitos pela instalação de barragems hidrelétricas em território Mapuche aumentaram nos últimos vinte anos. Assim, políticas que se propõem a incluir os povos indígenas também começam a ser implementadas. Neste artigo exploro como o multiculturalismo expresso na implementação da consulta indígena em projetos hidrelétricos no território Mapuche está relacionado às mudanças na política energética. Para isso, passarei a expor as linhas centrais de uma definição de multiculturalismo neoliberal extrativista. Em seguida, farei um relato da questão hidrelétrica no território Mapuche, incluindo a expansão das energias renováveis não convencionais, NCRE. Por fim, analiso da implementação da consulta indígena em projetos hidrelétricos no território Mapuche, especialmente revisando as dinâmicas de inclusão e exclusão ocorridas. Este artigo é baseado em minha pesquisa de doutorado em andamento com interesse em analisar o lugar da Nação Mapuche no espaço político em um contexto de conflitos pela hidroeletricidade. Metodologicamente, desenvolvo uma abordagem qualitativa indutiva que me permitiu observar primeiro o conflito social e, em seguida, identificar fenômenos específicos associados à política de reconhecimento.

Palavras-chave: multiculturalismo, consulta indígena, política enegética, barragems hidrelétricas, nação Mapuche

Desde comenzada la década de los noventa los conflictos por la instalación de proyectos hidroeléctricos en territorio mapuche han sido una continuidad. De los grandes proyectos de embalse que se iniciaron en los noventa, comenzaron a existir desde mediados de los dos mil una diversidad de proyectos de pequeñas centrales de pasada, los que también han sido cuestionados. Así también, se consolida la discusión sobre el reconocimiento de derechos y la existencia de los indígenas en tanto pueblos que debían ser incluidos en los diversos debates societales. Ello ha implicado que el Estado implemente una serie de medidas, como son la consulta indígena. Este artículo se basa en mi investigación doctoral en curso interesada en el análisis del lugar del Pueblo Nación Mapuche en el espacio político en el contexto de los conflictos por la hidroelectricidad. Mis datos corresponden a entrevistas realizadas a actores locales, trabajadoras y trabajadores del Estado y de ONGs, notas de trabajo de campo, así como documentos de los expedientes de cada consulta indígena (Servicio de Evaluación Ambiental, 2020), prensa y estudios de caso que se han desarrollado sobre proyectos específicos. Metodológicamente, me oriento por la teoría fundamentada y sus procedimientos de análisis (Strauss \& Corbin, 2004; Glaser \& Strauss, 1967; Soneira, 2006). Desde este enfoque cualitativo de carácter inductivo primero observé de manera abierta la conflictividad social y categoricé los primeros fenómenos asociados al relacionamiento político entre actores que discutían la aprobación de proyectos. En una etapa posterior, a través del muestreo teórico seleccioné tipos de conflictos asociados a la aplicación de la consulta indígena. Luego, implementé la estrategia de comparación constante, que me per- 
mitió analizar similitudes y diferencias en la implementación de la consulta indígena caso a caso. Me concentro en las dinámicas de inclusión/exclusión de las que son objeto los Mapuche que se encuentran en disputa con las empresas hidroeléctricas y el Estado. En esta etapa dialogué con la teoría existente, siguiendo la literatura sobre multiculturalismo y conflicto social. Entonces, en este trabajo me propongo presentar una de las discusiones derivada de los resultados de investigación: el multiculturalismo neoliberal extractivo expresado en la implementación de la consulta indígena en proyectos hidroeléctricos en territorio mapuche surge con los cambios de la política energética. En el escrito procederé a exponer elementos centrales de una definición de multiculturalismo neoliberal extractivo. Luego, daré cuenta de la cuestión hidroeléctrica en territorio mapuche, incluyendo la expansión de las energías renovables no convencionales, ERNC, fenómeno que ocurre en el marco de las transformaciones del sector eléctrico. Finalmente, analizo la implementación de la consulta indígena en los proyectos hidroeléctricos en el territorio mapuche, revisando especialmente las dinámicas de inclusión y exclusión que se han producido con la puesta en marcha de la política del reconocimiento.

\section{Multiculturalismo neoliberal extractivo}

\section{Multiculturalismo neoliberal}

El multiculturalismo en tanto idea comenzó a desarrollarse en Canadá y Australia en la década de los sesenta, principalmente para responder a las demandas de las minorías étnicas de estas sociedades fuertemente basadas en el flujo migratorio y la diferencia racial, oponiéndose a la asimilación de los inmigrantes a una sociedad nacional unitaria, cuestión conocida como melting pot (Armony, 2001: 1; Doytcheva, 2011: 5). Entonces, surgen de este debate las políticas multiculturales de reconocimiento que tienen como contexto los movimientos emancipatorios de la época, instalando una dinámica en que estos actores comenzaron a ser incluidos en la toma de decisiones públicas, dentro del control estatal (Kymlicka, 2013: 102-103). El reconocimiento como idea de raigambre hegeliana, supone la necesidad de afirmación de la otredad como base constituyente de la identidad, la importancia de las relaciones dialógicas ante la diferencia cultural y la gestión institucional basada en la autonomía cultural y política de las minorías nacionales y pueblos indígenas (Taylor, 1994: 52).

Entonces, en Norteamérica estas medidas reconocieron los derechos a la tierra y el autogobierno de los pueblos indígenas o Primeras Naciones; al fortalecimiento de la autonomía regional y el estado del idioma oficial para grupos nacionales subestatales, como es el caso de la provincia de Quebec en Canadá; y a adoptar más políticas de acomodamiento para grupos de inmigrantes (Kymlicka, 2015: 209). No obstante, la tradición liberal plantea que los derechos colectivos, incluido el autogobierno, se resguardarán a través de la consagración de derechos individuales (Kymlicka, 1995). Actualmente, estas políticas son fuertemente criticadas por las limitaciones que han significado para la autodeterminación de los pueblos indígenas, pues reproducen las configuraciones de poder estatales colonialistas, racistas y patriarcales (Coulthard, 2018: 17; Alfred, 2009: 133).

En América Latina, el multiculturalismo, se conoce en varios formatos desde mediados de los años 80 por ampliar la participación de pueblos indígenas y afrodescendientes (Postero, 2007: 
14). Estas transformaciones políticas deben comprenderse a la luz de la fuerza que las movilizaciones indígenas han tomado en la región, en especial después de los periodos dictatoriales, en los contextos de las transiciones electorales (Sieder, 2002). Incluso, en la región se ha configurado un multiculturalismo constitucional que crea marcos jurídicos pluralistas basados en el reconocimiento de derechos indígenas (Van Cott, 2000; Sieder, 2002).

Desde la década de los 70, con el surgimiento y consolidación de las políticas neoliberales, el multiculturalismo ha adoptado un carácter que ya no se orienta sólo al Estado y al fortalecimiento de la nación, sino que se hace parte de procesos globales. El neoliberalismo organiza la producción basándose en la competencia de mercado, y se diferencia del liberalismo pues asume que el mercado requiere ser construido a través del disciplinamiento del Estado sobre los actores privados (Maillet, 2015: 111-113). Por lo tanto, entiendo el neoliberalismo tanto como una doctrina económica como política, que también implica dimensiones culturales y simbólicas. Entonces, con la irrupción de nuevos actores y lógicas económicas neoliberales la gestión de la diferencia se requiere para mantener el flujo del capital global dentro y fuera del territorio (Mitchell, 2004: 123-124). Así, el avance del mercado global se sirve de la inclusión multicultural para incorporar a los Otros a las mismas dinámicas, fortalecerlas, así como neutralizar la diferencia y el conflicto, mientras las jerarquías raciales y la desigualdad persisten (Boccara, 2011; Hale, 2006; Postero, 2007; Richards, 2013; Žižek, 1998). Además, el multiculturalismo neoliberal está teniendo implicancias en la construcción de la subjetividad indígena. Silvia Rivera Cusicanqui propone el concepto de Indio Permitido, como aquel que reproduce la relación concordante con las propuestas multiculturales del Estado y de la sociedad mayoritaria (Cusicanqui, 2016: 67). A ésta se le opone el sujeto indígena que discrepa y sobre el cual recae el peso de la fuerza represiva formal y simbólica del Estado (Hale \& Millamán, 2005). En las secciones siguientes muestro cómo se impulsa un tipo de dialogo orientado por los requerimientos del mercado eléctrico y que se implementa con diversas limitaciones.

\section{Las lógicas de la inclusión y exclusión del multiculturalismo}

El multiculturalismo, para integrar e incluir a las minorías étnicas o grupos subordinados en determinado orden social, genera criterios que al mismo tiempo excluyen. Entiendo este fenómeno desde la teoría del discurso, para la cual hegemonía es principalmente resultado de prácticas de articulación entre sectores de la sociedad que intentan clausurar parcialmente un determinado orden, en relación con aquello que ha sido excluido de lo social (Laclau \& Mouffe, 2011: 177-178). Los mecanismos de inclusión y exclusión son fundamentales en el desarrollo de las prácticas hegemónicas pues clasifican aquello que le es externo a lo social, trazando fronteras que siempre tienen carácter flexible y contingente (Norval, 1994: 121). La dinámica inclusión/exclusión es parte del proceso de configuración de la identidad social: incluimos a través de cadenas de equivalencias aquello que permite constituirnos, y excluimos aquello que nos amenaza, que se opone al quienes somos, lo que los autores de esta corriente entienden, inspirados en Saussure, como lógica de la diferencia (Laclau \& Mouffe 2011: 174,187). Este fenómeno es abordado en términos relacionales, entendiendo que la constitución de la identidad se funda en una "exterioridad constitutiva" (Mouffe, 2007: 22). En tal sentido, la inclusión/exclusión va estipulando qué sentidos son los que se van fijando asociados a determinada identidad, y por lo tanto a lo social, y qué prácticas hegemónicas son las que la moviliza. Siempre existirá inclusión asociada a determinada forma de exclusión, pero ¿por qué se expresa una cierta forma de inclusión, asociada a 
determinada exclusión? Así, siguiendo a Lasse Thomassen (2017), el interés del estudio de estos mecanismos, dinámicas y prácticas está en la posibilidad de dar cuenta las relaciones de poder que fijan el adentro y el afuera. También, nos permite analizar las instituciones o espacios de inclusión, y si la exclusión resulta implícita o explícita. Finalmente, como toda práctica social, la inclusión/exclusión es contingente, temporal y relativa; y por lo tanto investigar sobre ello puede contribuir a cuestionar y modificar lo establecido. En definitiva, la identidad y la inclusión pueden ser estudiadas como resultado de las luchas hegemónicas por las representaciones (Thomassen, 2017: 6-7, 37,133).

En mi trabajo de investigación, he documentado un conjunto de prácticas hegemónicas observadas en políticas de Estado que han legitimado y ayudado a implementar el régimen colonial chileno para apropiarse del territorio ancestral mapuche o Wallmapu desde que lo ocupa militarmente en el siglo XIX hasta la fecha ${ }^{3}$. En particular, en este artículo analizo cómo las actuales formas de inclusión de los pueblos indígenas en el marco de las políticas del reconocimiento han trazado mecanismos que producen formas de exclusión que, desde lo que he estudiado, limita los procesos de toma de decisión sobre el territorio y su futuro. Así, el surgimiento de la política del reconocimiento en la política energética y en los procedimientos de toma de decisiones de proyectos extractivos lo comprendo en tanto resultado de lógicas de equivalencia, entre sectores gubernamentales y empresariales que impulsan el sector económico de la energía "verde". Éste requiere del multiculturalismo neoliberal para incluir a los sectores indígenas con la expectativa de disminuir la conflictividad social y consolidar entornos de aceptabilidad social de iniciativas. Al mismo tiempo, se generan una serie de exclusiones tanto de los espacios de participación y deliberación, como del acceso a la justicia ambiental. Por ello, la cualidad "extractivo" del multiculturalismo neoliberal se refiere al impulso específico que realizan las políticas del reconocimiento respecto a los proyectos de inversión que se basan en la mercantilización de los bienes naturales comunes. Ello se comprende desde un marco mayor, que es el extractivismo, modelo de desarroIlo que se caracteriza por la explotación de grandes volúmenes de materias primas, que dependen de economías de enclave y que se exportan como commodities (Svampa, 2012: 23; Gudynas, 2013: 3). La generación y distribución de energía hidroeléctrica en Chile la entiendo dentro de este modelo pues el agua es un commodity (Prieto y Bauer, 2012), la energía se requiere para la expansión de la exportación, y se proyecta su crecimiento con este mismo objetivo. En definitiva, en Chile, los ríos y las aguas se entienden como recursos estratégicos (Hernando, 2017: 136). En las secciones siguientes daré cuenta de cómo se fue instalando el multiculturalismo en Chile y cómo ello se expresa en la reciente expansión de la cuestión energética en territorio mapuche, en particular en el aumento de proyectos de pequeñas centrales hidroeléctricas.

\section{Dinámicas específicas del multiculturalismo neoliberal en Chile}

En Chile este tipo de políticas del reconocimiento multicultural se fueron instalando de la mano de la expansión neoliberal sobre los territorios en el periodo de la posdictadura. Éstas se han implementado de manera paulatina, e incluso tardía respecto de otras realidades latinoamericanas. Un hito de la relación política de la dirigencia de la centro-izquierda chilena con pueblos indígenas se da hacia el fin de la dictadura en el Encuentro de Nueva Imperial en 1989. Este

Una conceptualización de Wallmapu, territorio o país mapuche se encuentra desarrollada en el trabajo de Pablo Marimán (Marimán, 2006 : 54-58). 
evento sienta las bases de las políticas futuras del reconocimiento ya que, Patricio Aylwin, quien luego fue Presidente de la República se comprometió a reconocer a los pueblos indígenas en la Constitución, a generar una institución encargada de las políticas de desarrollo, promoción cultural y social, y a crear una comisión orientada a abordar las reivindicaciones indígenas para ser incorporadas en el plan de gobierno. Sin embargo, esta alianza no fue exitosa en términos de sus resultados. Se promulgó la llamada Ley Indígena, que creó la Corporación Nacional de Desarrolló Indígena CONADI (1993)4, que fue fuertemente criticada por sus limitaciones. Incorpora la gestión y compra de tierras y aguas, políticas de fomento económico, un reglamento asociado a la identificación indígena, entre otros. Sobre todo, esta legislación define a los indígenas como etnia, luego que se rechazara la inclusión de la expresión Pueblos Indígenas del proyecto que originalmente la Comisión Especial de Pueblos Indígenas -CEPI- había propuesto (Aylwin, 2002: 102). Además, la Ley Indígena creó un Consejo Indígena que incluye ocho representantes, siendo el único mecanismo de participación indígena que posee un carácter consultivo respecto a las decisiones del Estado (Aguas \& Nahuelpan, 2018: 5; Programa de Derechos Indígenas, 2003: 16). El reconocimiento constitucional indígena si bien se incorporó al debate parlamentario, hasta la fecha esto no ha sido aprobado.

En la década siguiente, en el 2001 se inaugura, la Comisión Verdad Histórica y Nuevo Trato con los Pueblos Indígenas, CVHNT. Ésta debía avanzar en la generación de políticas públicas a través de un proceso de diálogo en el que participaron una serie de organizaciones indígenas claves, así como una amplia gama de actores de la sociedad civil chilena: La Comisión se ha formado la convicción que no puede haber Nuevo Trato sin reconocimiento, y no puede haber reconocimiento sin que las voces silenciadas sean escuchadas" (Comisionado Presidencial para Asuntos Indígenas, 2008: 533). Finalmente, esta instancia no tuvo resultados de envergadura ni en lo práctico ni en lo simbólico. Más bien se ha criticado la contradicción que significó este llamado del gobierno de Ricardo Lagos a refundar la relación con los pueblos indígenas mientras se aplicaban lógicas fuertemente represivas, entre ellas la ley antiterrorista a los sectores mapuche movilizados contra las industrias forestal e hidroeléctrica (Aguas \& Nahuelpan, 2018: 7; Aylwin, 2005: 53-55).

En estos mismos años, comenzaron a elaborarse políticas inspiradas por el multiculturalismo en el plano de la educación y la salud. Diversos autores sitúan la experiencia del Programa Orígenes (2001-2010), iniciativa financiada por el Banco Interamericano de Desarrollo, como una de las formas concretas en que se pone en marcha una política de tipo multicultural de carácter neoliberal (Bello, 2007; Boccara \& Bolados, 2010; García Peter, 2016; Richards, 2013). El gobierno orientaba dichos fondos en territorio mapuche bajo una lógica asistencialista en zonas altamente movilizadas con el fin de desactivar los procesos de organización intentando contener y restringir las reivindicaciones, al mismo tiempo que se reprimía a los líderes locales (Bello, 2007).

Luego, en el marco del primer gobierno de Michelle Bachelet (2006-2010) acontece un nuevo empuje multicultural. El 2007 Chile vota a favor de la Declaración de Naciones Unidas sobre Derechos de los Pueblos Indígenas en la ONU y luego el 2008 se promulga la política Re-Conocer, Pacto Social por la Multiculturalidad. En este documento se entiende multiculturalidad como "la aceptación e inclusión de los pueblos indígenas en la sociedad chilena" (Gobierno de Chile,

Le 19253 Establece Normas Sobre Protección, Fomento y Desarrollo de los Indígenas, y crea la Corporación Nacional de Desarrollo Indígena 
2008: 11). Estamos frente a una política de carácter integracionista que tiene por foco la apertura a la diferencia que significan los indígenas desde un plano netamente cultural y económico. En efecto, un ámbito específico de esta política fue "Gestión del Desarrollo Integrado", uno de los inicios en el debate gubernamental sobre multiculturalismo y política extractiva, pues se proponía crear un código de conducta responsable para la implementación de proyectos de desarrollo en Áreas de Desarrollo Indígena y tierras indígenas. Aquí se insta al respeto de la consulta, a la distribución de beneficios, el derecho a la compensación por los daños causados y a la implementación del artículo 16 relativo al traslado de Pueblos Indígenas (p. 20). La proyección era que este código se incorporara en la legislación, sin embargo, estas medidas no fueron implementadas. Este mismo año se ratificó el Convenio 169 de la OIT sobre Derechos de los Pueblos Indígenas y Tribales, C.169, luego de 18 años de debate parlamentario. Con ello se abrió una disputa respecto a la implementación de la Consulta Indígena, ya que este Convenio explicita este derecho, Así, posteriormente y luego de varios debates fue reglamentada y puesta en práctica en contextos de evaluación ambiental de proyectos de inversión desde el 2013. También comenzaron a abrirse varias unidades de asuntos indígenas que comenzaron a crear políticas sectoriales específicas, tal como más adelante lo realizó el Ministerio de Energía.

Así, el multiculturalismo en Chile se ha centrado en la redistribución económica y cultural pero no en una redistribución política (Richards, 2013). En suma, esta relación entre multiculturalismo y reconocimiento está cuestionada por los límites que enfrenta al momento de definirse el espacio político para que los pueblos indígenas definan autónomamente su avenir. Los contextos extractivistas son todavía un escenario más proclive para que esta tensión se manifieste: los indígenas son actores políticos de los espacios geográficos que requiere el capitalismo global para su expansión. Por ello no es un hecho fortuito que el fortalecimiento del extractivismo en Chile haya estado asociada a la discusión sobre los derechos indígenas y la política del reconocimiento multicultural.

\section{Hidroelectricidad en Chile}

\section{Hidroelectricidad en territorio Mapuche}

La hidroelectricidad es uno de los sectores de la economía política del país donde se concentran conflictos socioterritoriales (Allain, 2019; Delamaza, Maillet \& Martínez Neira, 2017). En las regiones del centro sur del país, la mayor parte de los casos asociados a estas inversiones se sitúan en tierra y territorio mapuche, y las organizaciones, lof (o agrupaciones tradicionales) y comunidades son actores sociales preponderantes (Tironi \& Pircović, 2017; Instituto Nacional de Derechos Humanos, 2015). En Chile, hacia el fin del siglo XIX la hidroelectricidad comenzó a desarrollarse para fortalecer la producción minera y comenzar a dotar de energía las grandes ciudades (Yañez, 2017a; Napadensky Pastene, 2007). En este periodo avanzaba la ocupación militar que concretó el despojo del que fue objeto el Pueblo Nación Mapuche luego de la violenta ocupación del Estado que impuso una forma de propiedad, fomentó la ocupación de colonias europeas y redujo a la población mapuche en mínimas cantidades de tierra (Marimán, 2006; Giminiani, 2015).

En la fase siguiente a la ocupación, la consolidación de las actividades económicas y de las ciudades incluyó la instalación de centrales hidroeléctricas desde inicios del siglo XX. Correspon- 
den a pequeñas obras privadas gestadas para el servicio de electricidad pública de localidades emergentes y el autoabastecimiento para las actividades agrícolas. Tal es el caso de la central Llollelhue (1909), en La Unión (Vásquez, Valdebenito \& Ogalde, 2018: 5-6) y las centrales Loncoche y Gorbea (década de los treinta) (Yañez, 2017a: 182). Más adelante, cuando las ciudades del centro sur del país y las actividades industriales se complejizaron, se inician las primeras políticas eléctricas de Estado en materia energética con la creación de la CORFO en 1939, que a su vez creó la Empresa Nacional de Desarrollo Eléctrico Sociedad Anónima, ENDESA en 1944. Esta empresa, que luego llegó a monopolizar el sector eléctrico decidió emprender una serie de obras a lo largo del país (Yañez, 2017b). En particular, entre las actuales regiones administrativas del Bío Bío y Los Lagos se construyeron cinco centrales: la Central Pilmaiquen en 1944; la central Pullinque en 1963 y las centrales Abanico en 1948, El Toro 1973 y Antuco 1981.

Sobre la vida mapuche en los entornos de estas centrales no se encuentran registros que se refieran a ello sino hasta los últimos años. En el caso de ENDESA, en el material publicitario de cada una de las infraestructuras, así como en los informes de CORFO no se presentan estos territorios como espacios habitados. Actualmente, han surgido diversas reivindicaciones asociadas a los efectos de dichas centrales y los despojos que implicaron, en particular respecto a las centrales Pilmaiquén y Pullinque, ambas de propiedad de Enel Green Power. Actualmente, la comunidad mapuche Mapu Pilmaiquén reivindica su territorialidad en el entorno de la central Pilmaiquén. A causa de esta demanda, la empresa reconoció que los miembros de dicha comunidad habitan la zona desde hace más de un siglo y les cedió un espacio en comodato para la administración de un parque (ENEL, 2019). Sin embargo, actores locales explican que la ocupación de este espacio es de tipo ancestral, que han continuado con prácticas culturales y espirituales sobre este lugar (González \& Molina, 2019). También en torno a la central Pullinque existe una reivindicación territorial a causa de la inundación de las tierras mapuche que dejó inutilizadas cerca de 600 hectáreas y otras que fueron secadas a causa de la desviación de $4,5 \mathrm{~km}$ del rio Huenelhue que fue conducido por un canal (Consejo de Comunidades Pullinque, 2017). Algunas comunidades han acordado un marco de compensaciones con la empresa, pero otras persisten en el desacuerdo reclamando reconocimiento de afectaciones materiales e inmateriales, compensaciones justas y restauración del caudal ecológica del río (Ibarra Eliessetch \& Riquelme Maulén, 2019; Collinao, 2014; Asociación Leufü Wueneywue, 2018).

El Estado empresarial, gestor de la electricidad terminó en el periodo dictatorial, momento en el se privatizó la mayor parte sus empresas en el sector eléctrico y se creó la Ley General de Servicios Eléctricos (1982). Entonces, se consolidó una política neoliberal de carácter tradicional, sentando las bases para la competencia entre actores privados que buscaban ganancia a corto plazo (Maillet \& Rozas Bugueño, 2019: 214). Iniciado el periodo posdictatorial se reactivó la construcción de grandes embalses en el centro sur de Chile. Las primeras fueron centrales en el Alto Bío Bío en territorio mapuche-pehuenche, Pangue y Ralco, construidas en un contexto de conflicto social de envergadura que marcó la posterior relación del sector hidroeléctrico con las organizaciones del movimiento mapuche (Opaso, 2012; Orellana, 2005). Posteriormente, a mediados de los dos mil, se conocieron nuevas ideas de grandes empresas que igualmente fueron fuertemente contestadas: las centrales de la noruega SN Power en Liquiñe y Maqueo; de Endesa (hoy Enel) en Neltume; Angostura y San Pedro de Colbún, y las centrales Osorno y Los Lagos de Strakraft en la cuenca del Pilmaiquén. De todos estos proyectos sólo se construyó Angostura. La Central Los Lagos inició su 
construcción el 2019, pero persiste el conflicto social, y continua la evaluación de la central San Pedro. Los otros fueron desistidos por sus titulares.

\section{La expansión de las ERNC}

Desde mediados de los dos mil comienza un proceso gubernamental de modernización del sector eléctrico con un proyecto de ley que enfrentó los problemas de organización y regulación (Biblioteca del Congreo Nacional de Chile, 2004: 7). Así se consolidaron en la última década una serie de transformaciones en las normativas, las políticas y el mercado eléctrico cuestión que da cuenta de una hibridación neoliberal en la política energética motivada principalmente por la dinamización competencia (Maillet y Rozas Bugueño, 2019: 230). Esto ha tenido implicancias concretas en el desarrollo de conflictos por la instalación de proyectos energéticos en el Wallmapu. De la conflictividad por los grandes embalses hidroeléctricos surgieron los conflictos con centrales de pasada ya que se incentivó el sector de las energías renovables no convencionales, ERNC.

Dos debates son centrales cuando se inician estos cambios: la necesidad de asumir la diversificación de la matriz energética para cumplir con las obligaciones frente al Protocolo de Kyoto y la independencia energética (Tokman, 2008: 51, 97). Además, aunque todavía no era un asunto central, se comienza a problematizar la implementación del C.169 como un asunto de viabilidad política y social del desarrollo energético (Tokman, 2008: 91-91). La primera ley para el desarrollo de las ERNC, la Ley 20.257 del 2008 estableció la obligación de las empresas eléctricas que comercializan energía en los sistemas eléctricos con capacidad instalada superior a $200 \mathrm{MW}$ de inyectar 5\% de ERNC a los sistemas eléctricos (Tokman, 2008: 80) y definió las ERNC ${ }^{5}$. En este contexto se suma al debate el enfoque ambiental sobre los efectos de los grandes proyectos energéticos. También, el 2011 reportes especializados coincidieron en el diagnóstico de que el mercado eléctrico tenía una alta concentración de la oferta, por lo que había un déficit de competencia; que existía alta resistencia ciudadana a los proyectos de generación; y que era urgente reducir la emisión de gases de efecto invernadero (Maillet \& Rozas Bugueño, 2019: 223) Así, el primer gobierno de Piñera (2010-2014) promulgó la Ley 20.698, conocida también como Ley 20/25, que fijaba que el objetivo obligatorio del mercado fuera de un 20\% de ERNC al 2025 (Rudnick \& Palma, 2018: 28). Además del apoyo a las ERNC, este gobierno hizo explícito su intención de fortalecer la hidroelectricidad debido a alto potencial hídrico del país (Ministerio de Energía, 2012: 9).

La crisis de precios y la movilización ciudadana vendría a consolidar aún más las ideas que defendían la presencia del Estado en el sector eléctrico, así como el impulso a las energías sostenibles y a las medidas de planificación que consideraran una dimensión participativa. Así, durante el segundo mandato de Michelle Bachelet (2014-2018) se concretó la primera política energética de Chile denominada Energía 2050 (Ministerio de Energía, 2015) que, junto a un cuerpo normativo, consolidó el rol del Estado como promotor del mercado eléctrico. En definitiva, el viejo modelo de política energética basado en del mercado autorregulado, cambió definitivamente a uno en que la presencia del Estado es vital para incentivarlo, aumentar la competencia y asegurar la infraestructura. (Madariaga \& Gladina, 2018: 35-36; Maillet \& Rozas Bugueño, 2019: 229-231).

Energías renovables no convencionales (ERNC) corresponderán, según esta norma, a centrales hidroeléctricas pequeñas (menos de 20 MW de potencia máxima), y a proyectos que aprovechen la energía de la biomasa, la hidráulica, la geotérmica la solar, la eólica, de los mares, entre otras. 
Según el impulsor de esta política, el ministro de energía Máximo Pacheco, el quehacer del Estado debe ser en torno al manejo de los precios y esto se logra en base a la desconcentración de los mercados, la diversificación de la matriz energética con más proyectos de ERNC y el control de los conflictos sociales para que los proyectos se construyan (Pacheco, 2018). En definitiva, desde este enfoque, el bajo precio de la energía es lo que garantiza el bien común (Nuñez, 2018: 489), sin que se hayan cuestionado los principios neoliberales que lo rigen.

Así, en Energía 2050 se propuso que al 2035 40\% de la energía provenga de este tipo de fuentes. Una acción que se destacó fue el Plan 100 Mini-Hidros. El objetivo era llegar a la implementación de 100 proyectos de pequeñas centrales Hidroeléctricas, es decir, de generadoras de menos de 20MW. 70"\% de estas inversiones se ubicaban entre el Maule y Araucanía (Nuñez, 2018: 491). En específico, Pacheco anunció que 18 centrales hidroeléctricas serían construidas en la región de La Araucanía (Álvarez 2015, p. 14; Soto 2015), cuestión que alertó a las organizaciones locales las que incluso llegaron hasta el parlamento (Mapuexpress, 2016). Al finalizar el mandato de Bachelet, en diciembre de 2017, existían 66 centrales ejecutadas, de las cuales 54 estaban en operación y 12 en construcción, aumentando la capacidad instalada de generación eléctrica en 77\% (Nuñez, 2018: 493, 501).

En definitiva, han aumentado las ERNC en el área comprendida como el Wallmapu, que de norte a sur implica las regiones del Bío Bío, Araucanía, Los Ríos y Los Lagos. Según los datos (Comisión Nacional de Energía, 2020), en estas regiones, se genera 48,8\% de la hidroelectricidad total del Sistema Eléctrico Nacional, SEN, y se ubica el $47.8 \%$ de la capacidad instalada de las pequeñas centrales de pasada, que son las catalogadas como ERNC. Éstas comenzaron a operar desde 1995, sin embargo, aumentan desde el 2011. Cabe destacar que la Región de la Araucanía no contaba con ningún tipo de central hidroeléctrica en operación hasta el 2008. En esta región, sólo existen pequeñas centrales hidráulicas, que se contabilizan en 15 unidades. Otro tipo de energía renovable emergente en este macroterritorio es la eólica, que representa el $12 \%$ de la capacidad instalada en esta zona y que comenzaron a operar el 2014.

En consecuencia, este nuevo enfoque del Estado como promotor del sector ERNC ha significado el surgimiento de nuevas inversiones, nuevos actores empresariales, y nuevas dinámicas de relacionamiento con la comunidad local. En efecto, la política inspirada en las experiencias mineras también fomenta mecanismos de asociatividad y diálogo con la comunidad (Ministerio de Energía, 2016); un componente de planificación participativa como mecanismo de legitimación social de las decisiones ministeriales; y generó la primera mesa de trabajo con pueblos indígenas en materia de energía que dio pie al Capítulo Indígena de la Política Energética (Ministerio de Energía, 2017). Este marco discursivo de integración de los pueblos indígenas a la planificación del desarrollo eléctrico se estructuró en paralelo al aumento de conflictos y proyectos que estaban siendo analizados por el Servicio de Evaluación de Impacto Ambiental, SEA, organismo a cargo de otorgar las licencias ambientales de proyectos extractivos y que está a cargo de implementar la consulta indígena en este marco. El segundo gobierno de Sebastián Piñera (2018 en adelante) ha continuado con las directrices de la política energética diseñada en el gobierno de Bachelet, ampliando las perspectivas de fomento de las ERNC al conjunto de energías de este tipo, considerando que el sector creció y se diversificó más de lo esperado. En la sección siguiente analizo la implementación de la consulta indígena en el sector hidroeléctrico, medida del reconocimiento 
multicultural que está ejecutando el Estado para consolidar el dialogo con las comunidades indígenas, aunque veremos, su alcance es extremadamente limitado.

\section{Multiculturalismo neoliberal extractivo en los conflictos hidroeléctricos en territorio Mapuche}

Me interesa dar cuenta de las relaciones de poder que se configuran con el diseño e implementación de la política del reconocimiento en el plano de los conflictos socioterritoriales por las inversiones hidroeléctricas que se proyectan en territorio mapuche. Conforme a mi metodología, la Teoría Fundamentada, en una primera etapa identifiqué que en torno a la política del reconocimiento y la consulta indígena existía un tipo de conflictividad que merecía una observación específica. Ello me permitió profundizar respecto de este debate político nacional. El fenómeno que identifiqué en esta etapa fue el "enmarcamiento de la consulta indígena" cuestión que influye y define cómo se desarrollan varios de los conflictos específicos. En una segunda etapa apliqué un muestreo teórico, que es la selección de los casos respecto a sus atributos e implementé la estrategia de la comparación constante. Este método propone construcción de teoría a partir de la búsqueda de similitudes y diferencias en los datos (Glaser \& Strauss 1967: 103). Así, analicé los 7 procesos de consulta indígena realizadas para proyectos hidroeléctricos, así como otros 9 casos en que no se ha implementado y donde existía o existe conflicto por esto. Luego, establecí preguntas que orientaron un proceso de codificación en torno al fenómeno central, las dinámicas de inclusión y exclusión ¿Cómo se ha fijado el adentro y el afuera de la relación del Estado con las comunidades, lof y familias mapuche concernidas por los proyectos hidroeléctricos que se proponen? ¿Qué ha significado la inclusión en la deliberación de los proyectos propiamente tal para las comunidades mapuche? ¿Cómo interactúan las comunidades ante los marcos inclusivos, pero también frente a la exclusión que se ha ido desarrollando en la toma de decisiones de los asuntos energéticos e hidroeléctricos en particular?

\section{Enmarcamiento de la consulta indígena como medida del multicultu- ralismo neoliberal}

La primera medida de reconocimiento multicultural que tiene implicancia directa con los conflictos por la instalación de proyectos extractivos se da en el marco de la ratificación del C.169. Específicamente, este tratado incluye el derecho a la consulta indígena, una herramienta específica de participación política que debe estar garantizada por el Estado (Meza-Lopehandía Glaesser, 2016: 22). Estos procedimientos pueden terminar sin acuerdos o sin la obtención del consentimiento, salvo cuando las medidas que adopten los Estados pongan en riesgo la existencia de una cultura indígena (Millamán Manríquez, 2019: 6). En un primer momento, el gobierno redactó una norma, el decreto 124 de 2009, que descartó aplicar este derecho en la evaluación de proyectos extractivos, que luego fue derogado con una nueva norma, el Decreto 66 de $2014^{6}$ (Silva Neriz, 2014; Carmona Caldera, 2013).

Decreto 66. Aprueba reglamento que regula el procedimiento de consulta indígena en virtud del artículo $6 n^{0} 1$ letra a) y no 2 del Convenio no 169 de la Organización Internacional del Trabajo y deroga normativa que indica. Disponible en: https://www.leychile.cl/Navegar?idNorma $=1059961$ 
Finalmente, fue en el marco de la normativa ambiental que el derecho a la consulta indígena se implementó en relación con los proyectos que ingresan al Sistema de Evaluación de Impacto Ambiental, SEIA. Específicamente, el Decreto Supremo 40 del 2013 que reglamenta el SEIA, RSEIA7, especifica en su artículo 85 que se aplicará la Consulta Indígena a los proyectos que ingresen al sistema de evaluación ambiental, en el caso que se afecte directamente a los pueblos indígenas y que se refieran a situaciones de "Reasentamiento de comunidades humanas, o alteración significativa de los sistemas de vida y costumbres de grupos humanos", a "Localización y valor ambiental del territorio" y "Alteración del patrimonio cultural". Así, se establece un requisito para la procedencia de la Consulta Indígena relativa a la dimensión de la afectación (Guerra Schleef, 2017: 23). También, el RSEIA indica que "deberá efectuarse con la finalidad de llegar a un acuerdo o lograr el consentimiento. No obstante, el no alcanzar dicha finalidad no implica la afectación del derecho a la consulta". Con ello, la normativa deja en claro que el proceso no es vinculante y que el consentimiento no es el objetivo de la consulta indígena, sino la deliberación en sí misma. En consecuencia, existe consulta indígena cuando el SEA, organismo a cargo del SEIA dictamine que existe susceptibilidad de afectación directa de comunidades indígenas a partir de la información entregada por el titular de un proyecto, cuestión que ocurre sólo cuando la envergadura de una inversión, según se define en la ley ambiental, le obliga a ingresar como Estudio de Impacto Ambiental. Por lo tanto, no habrá Consulta Indígena cuando procede una Declaración de Impacto Ambiental, y los impactos no se ponderen como significativos y la afectación no se considere directa. En el caso de las centrales hidroeléctricas, según esta norma sólo ingresan a evaluación los proyectos que generan más de $3 \mathrm{MW}$. Entonces, las centrales hidroeléctricas que declaran menor capacidad de generación no se ven sometidas a ningún procedimiento de información, consulta o participación ciudadana que discuta sus efectos ambientales y sociales (Hernando, 2017: 136; Kelly, 2019: 226). En definitiva, la cantidad de procedimientos de consulta indígena es mínima, y cuando se aplica, se restringe la participación a las comunidades que el SEA considera son las afectadas directas.

Además, el contenido de la deliberación ha sido limitado a los impactos significativos reconocidos por el titular y, por lo tanto, la consulta se refiere a las medidas de mitigación, reparación o compensación que propone una empresa respecto de su proyecto (Director Ejecutivo Servicio de Evaluación Ambiental, 2016: 10-13). Todo ello definido desde el punto de vista del organismo gubernamental, lo que ha generado una disputa en los tribunales por parte de comunidades que quieren hacer valer el derecho a la consulta. A la fecha el SEA informa que sólo se han desarrollado 56 consultas indígenas en todo el país y sólo 7 en relación con proyectos hidroeléctricos. Esta es una cifra baja considerando que sólo en la región de La Araucanía, existen 59 proyectos hidroeléctricos conocidos por el SEA desde el 2013, año en que se inicia la consulta indígena, a través de las figuras de consulta de pertinencia, DIA y EIA ${ }^{8}$. En definitiva, se ha configurado un enmarcamiento unilateral por parte de la sociedad dominante respecto de la consulta indígena, subestimando su valor político en el proceso decisional de los proyectos al restringir los actores a los que alcanzará, así como su contenido. Por lo tanto, esta imposición tiene un carácter colonialista al estar los pueblos concernidos excluidos del discernimiento de lo que se definió como criterios

Decreto 40. Aprueba Reglamento del Sistema de Evaluación de Impacto Ambiental. Disponible en: https://www.leychile.cl/Navegar?idNorma=1053563.

\& Esta cifra se obtiene a partir de la consulta de los datos públicos dispuestos por el SEA para proyectos ingresados al SEIA y para proyectos que se ingresan al sistema de Consultas de Pertinencia. Disponibles en https://seia.sea.gob.cl/ y en https://seia.sea.gob.cl/pertinencia/buscar. php. Consultado el 23 de marzo 2021 
de implementación de la consulta, de lo que es un impacto y su grado de significancia. Se confirma con esta medida de reconocimiento multicultural el lugar marginal que tienen los pueblos indígenas en el espacio político en Chile que define el desarrollo de los proyectos de inversión. Además, se evidencia el carácter neoliberal de la política que le otorga un rol preponderante a las empresas con sus estudios sobre los impactos, en desmedro de las comunidades indígenas que claramente no cuentan con las condiciones necesarias para disputarlos.

\section{Implementación de la consulta indígena en proyectos hidroeléctricos en Territorio Mapuche}

Las siete consultas indígenas realizadas a proyectos hidroeléctricos se localizan en territorio mapuche. Dos de estos siete proyectos corresponden a energía convencional y cinco a ERNC. Analicé las semejanzas y diferencias de los casos en relación con las dinámicas de inclusión y exclusión desarrolladas por el Estado. Evidencio que las características de los procedimientos varían bastante entre sí, dependiendo de las condiciones que exigieron o negociaron las comunidades participantes. Me baso en los documentos presentes en los expedientes públicos de cada evaluación ambiental, los que incluyen actas de cada reunión realizada por el SEA, protocolos metodológicos, informes de cierre de procesos, reportes específicos elaborados por las comunidades, declaraciones y cartas enviadas. También realicé 24 entrevistas, asistí a 6 eventos públicos sobre evaluación ambiental, 3 sobre política energética y recopilé material de política institucional, de prensa, y artículos académicos sobre cada consulta indígena.

Durante el 2013, los primeros tres proyectos hidroeléctricos en ser sometidos a Consulta Indígena fueron el proyecto Central Hidroeléctrica Neltume (490 MW) y el proyecto MiniCentral hidroeléctrica Florin II y Florin III (17,6 MW), ubicados en la región de Los Ríos; en la región de La Araucanía se sitúa el Proyecto Central Hidroeléctrica Añihuerraqui (9 MW). Luego, el 2015 se realiza para el proyecto Central Hidroeléctrica Rucalhue (90 MW) en la región del Bío Bío; el 2016 se inicia para los proyectos El Rincón (11 MW) en Araucanía y el 2017, comienza en Los Aromos (19.2 MW) y Hueñivales (15 MW). La consulta comenzó a operar desde el 2013 siendo el SEA la entidad a cargo y la empresa titular la responsable de entregar informaciones técnicas respecto al proyecto, aunque su implicación en las reuniones fue diferente según los requerimientos de las comunidades participantes. Se realiza una primera etapa de acuerdo metodológico entre el SEA y las comunidades que se consideraron como las afectadas, una etapa de información del proyecto y de reuniones deliberativas en que se discuten los impactos significativos y las medidas propuestas por la empresa, hasta llegar a la firma de un acta de acuerdos y/o desacuerdo de la comunidad con lo planteado. La duración de los procedimientos varió entre 28 meses en el caso más extenso, que fue respecto al proyecto Central Neltume -donde las comunidades que rechazaban el proyecto finalmente desistieron de continuar porque no se les aseguró el respeto del consentimiento (Cuadra Montoya, 2016)- y 6 meses en el caso menos extenso, que fue el proceso implementado en el proyecto central Rucalhue. Además, en varios casos se desarrollaron consultorías especializadas a petición de las comunidades locales, que fueron decididas por las comunidades participantes y financiadas por el SEA. Los objetivos de dichas asesorías divergieron según los intereses de las comunidades locales. Así, comunidades participantes de la consulta indígena pero opositoras a los proyectos demandaron consultorías de abogados y antropólogos que fortalecieron los argumentos técnicos de la oposición, como fue en los casos de Añihuerraqui, el Rincón y los Aromos. En otros casos se realizaron consultorías productivas o de asesoramiento cultural. 
En estos procesos deliberativos, las dinámicas de inclusión de las comunidades, lof y familias mapuche y sus resultados fueron distintos unos de otros. Observo que en los contextos en que existió aprobación de familias y comunidades participantes de los proyectos se desarrolló una negociación que llegó al acuerdo de las propuestas de las empresas. En los siete proyectos existieron comunidades que llegaron a acuerdo con el SEA por las propuestas de las empresas, aunque, en varios casos estas comunidades eran minoritarias o sólo una parte de las comunidades participantes de la Consulta Indígena. Está documentado para los proyectos Añihuerraqui y Neltume que las empresas negociaron anteriormente con familias y comunidades que estuvieron a favor del proyecto, facilitando la división y conflicto interno (Peralta, 2015; Höhl, 2020; Cuadra Montoya, 2014). Al momento de realizarse la evaluación, sólo en el caso del proyecto Rucalhue se observa acuerdo de todas las comunidades sin que existiera un proceso de movilización en oposición al proyecto. Al contrario, en los casos en que existía controversia un tipo de inclusión que se desarrolló fue de manera contenciosa (Roseberry, 2002), ya que las comunidades afectadas directamente participaron demarcando el contenido y la dinámica deliberativa: desarrollaron encuentros políticos o trawün, actividades propias de su cosmovisión, limitaron las instancias de encuentro con las empresas, y rechazaron negociar compensaciones, para finalmente, estipular desacuerdos en las actas de finalización. Esto se dio en los casos de los proyectos Anihuerraqui, El Rincón y Los Aromos. Además, cabe destacar que, en el caso de El Rincón, el titular en una primera instancia descartó la afectación directa y es luego de la movilización que el SEA reconoce a las comunidades de un macro territorio como las afectadas directas. En efecto, el río que se intervendría, el Truful Truful es un sitio de relevancia medicinal, cultural y espiritual para el conjunto de territorios y no sólo de las comunidades aledañas al río (entrevistas del 03-04-2018, 23-04-2018).

Otra situación es la exclusión de comunidades de los procesos de consulta indígena por no haber sido éstas consideradas afectadas directas, pero que igualmente se han movilizado, tal como sucede con los proyectos Centrales Florin II y III y Hueñivales. En el caso de las Centrales Florin II y III una de las comunidades del lof implicado y organizaciones territoriales denunciaron que el proceso de consulta indígena se encontraba viciado y que no se había considerado al conjunto de comunidades del Lof Huequecura (Kelly, 2018: 120-124). Éstas presentaron un reclamo administrativo ante el SEA por esta situación, pero que no fue acogido (Comisión de Evaluación Región de los Ríos, 2017). Respecto a la Central Hueñivales existe un proceso de reclamación abierto por la Comunidad Benancio Huenchupan que alegó haber sido excluida de la consulta siendo que son parte del territorio ancestral afectado (Madrid Meschi, 2019). También, una situación de exclusión, pero que finalmente se revirtió a inclusión se da en el contexto del rechazo inicial de empresas a considerar impactos significativos hacia las comunidades mapuche en las primeras etapas de los EIA. Sólo incorporaron a comunidades indígenas al área de afectación directa por la presión del SEA, los informes de CONADI y las observaciones de las propias comunidades, como se dio en el caso de Rucalhue y El Rincón.

Finalmente, del conjunto de proyectos con Consulta Indígena, sólo en dos ha existido una decisión final de rechazo en el proceso de evaluación ambiental: El Rincón ${ }^{9}$ y Los Aromos. Los

Inicialmente, El Rincón fue rechazado por la Comisión de Evaluación Regional de La Araucanía, el 22 de enero 2018. Sin embargo, el Comité de Ministros del Medio Ambiente revirtió dicho resultado, aprobando el proyecto, luego de un recurso de reclamación interpuesto por el titular. Actualmente, el proyecto se encuentra judicializado en el Tercer Tribunal del Medio Ambiente por parte de las organizaciones locales que esperan se respete la decisión de rechazo. 
argumentos utilizados por los agentes decidores estuvieron directamente asociados a los que construyeron las comunidades opositoras en el proceso de Consulta Indígena. Por el contrario, en el caso de Añihuerraqui, que se planteó como modelo de metodología de la Consulta Indígena, que este proyecto se hubiera aprobado existiendo argumentos tan sólidos en contra como es que se afectaría directamente el ngillatuwe o lugar de la ceremonia mapuche del territorio significó frustración y descrédito hacia la política del reconocimiento (entrevista con funcionario público 5-07-2018 y con un vocero mapuche local 5-06-2018). Este caso junto a otros proyectos aprobados corrobora que la decisión en manos de representantes de gobierno es política y no técnica, cuestión que se ha ampliamente discutido en el marco de la otorgación de permisos ambientales.

En relación con la implementación del diálogo y asociatividad de las empresas con las comunidades locales que llegaron a acuerdo en el proceso de consulta indígena, cabe destacar que éste se limita a un acuerdo de compensaciones más que a una distribución de beneficios que significara una participación de las comunidades y sus autoridades como actores del ciclo de los proyectos. Se identifican medidas compensatorias en el plano de lo social como mejoramiento de viviendas, sedes, escuelas o centros de salud; económicas, a través de becas o fondos, y culturales; como mejoramiento de infraestructura y equipamiento para las ceremonias. Las comunidades opositoras a estas medidas criticaron no sólo que no eran suficientes en relación con los beneficios económicos que las empresas obtenían, sino que no subsanaban impactos inmateriales que eran irreparables, pues en todos los casos existen impactos sobre los espacios naturales que son sitios de alto significado cultural y espiritual para el desarrollo de sus modos de vida (entrevistas del 07-07-2018 y 13-08-2018).

Entonces, dos hallazgos de mi análisis, realizado desde un enfoque inductivo-cualitativo, la Teoría Fundamentada, y basándome en evidencia empírica asociada a la puesta en práctica de la política del reconocimiento en la evaluación ambiental, permiten evidenciar la interrelación entre procesos estructurantes de la política del reconocimiento con procesos políticos localizados en el entorno de los proyectos hidroeléctricos. De una parte, constato que la consulta indígena, una expresión de la política del reconocimiento que busca incluir a las comunidades mapuche en los procesos de evaluación de impacto ambiental, se lleva a cabo bajo una forma de exclusión concreta derivada del enmarcamiento de este derecho en el plano jurídico y de la política. Se han definido criterios que delimitan a cuales actores se aplica la consulta indígena, definiendo cómo ésta será implementada por los sectores gubernamentales que se hacen cargo de la evaluación ambiental. De otra parte, este enmarcamiento es contestado por actores sociales que resultaron ser excluidos, pero también desde sectores incluidos y que terminan utilizando la consulta indígena de manera contenciosa para manifestar oposición a proyectos. Principalmente, estos últimos desarrollan una agencia que modifica y adecúa los términos de la relación que establecen con el Estado y las empresas, como es lo que ocurrió con comunidades afectadas por los proyectos Añihuerraqui, El Rincón y Los Aromos. De este modo, la política del reconocimiento si bien ha sido diseñada y dirigida jerárquica y unilateralmente, éstas últimas experiencias muestran que organizaciones mapuches han asumido la consulta indígena aplicando los principios de autonomía y libre determinación mapuche, redireccionándola según sus propios objetivos. Además, cabe mencionar la exclusión de las comunidades y territorios donde el SEA, a partir de la información que entregan los titulares en sus estudios, no confirma la existencia de impactos significativos y por lo tanto no abre el proceso de consulta indígena. Estos casos son muchos y varios de éstos se han judicializado, algunos emblemáticos como Doña Alicia, Mediterráneo y Llancalil que fue- 
ron aprobados en un primer momento, pero luego fueron revocados sus permisos por la justicia, justamente porque el SEA no realizó una labor suficiente para descartar la existencia de una afectación directa (Millamán Manríquez, 2019; Guerra Schleef, 2017).

\section{Conclusión}

La política del reconocimiento multicultural implementada recientemente en Chile luego de la ratificación del C.169 y las transformaciones en materia energética se relacionan estrechamente. De una parte, la hibridación neoliberal de la política de energía, como lo definen Maillet y Rozas Bugueño (2019), que se expresa a través de fortalecimiento del Estado en la dinamización del mercado, refuerza el sector de las Energías Renovables No Convencionales y con ello nuevos proyectos y actores en el territorio mapuche. Así, desde mediados de los años 2000 observamos un aumento de pequeñas centrales hidroeléctricas en el territorio mapuche, en paralelo al aumento de la conflictividad en este sector. De otra parte, se ha implementado la política del reconocimiento, con la consulta indígena y las instancias específicas de participación de los pueblos indígenas en la política energética, para gestionar el conflicto social.

En el análisis de la implementación de los procesos de consulta indígena en proyectos hidroeléctricos constato que se ha enmarcado restringiendo actores, territorios y proyectos de los procesos deliberativos. También, el diálogo con las organizaciones se limita a contenidos de los proyectos, sin que sea una instancia vinculante. Además, la retórica de la asociatividad, en tanto estrategia de inclusión, se concreta principalmente a través de compensaciones de parte de los empresarios para atenuar los impactos, pero no se han desplegado, relaciones concretas de distribución de beneficios. Estas son prácticas donde la posición de poder es desigual. Las empresas deciden qué negociar, la información es limitada, entre otros aspectos que son propios de las lógicas multiculturalistas en contexto neoliberal: incluir a los otros a las lógicas del modelo económico desde su particularidad cultural, manteniendo intacta la condición de subordinación en las relaciones de poder. Es decir, el Estado alimenta una subjetividad indígena condescendiente con las jerarquizaciones coloniales, el Indio Permitido, como lo conceptualiza Rivera Cusicanqui (2008). Por ello, el límite del multiculturalismo se hace evidente pues no ha calmado la movilización y el conflicto. Así, existe una interesante experiencia aquí en el Wallmapu para cuestionar este modelo como aquel que regirá una suerte de paz reconciliada entre pueblos indígenas y los Estados. Sobre todo, la consulta indígena como mecanismo de la política del reconocimiento no ha modificado la frontera identitaria que configura el antagonismo colonial mapuche-no mapuche en Chile, puesto que el dialogo que impulsa se organiza unilateralmente en beneficio del sector eléctrico. Futuras investigaciones podrían observar los impactos de la politización de las comunidades que deciden participar de las consultas indígenas de manera contenciosa, y si esto amplía la frontera de las relaciones políticas entre pueblos.

\section{Referencias bibliográficas}

AGUAS, J. y NAHUELPAN, H. Los límites del reconocimiento indígena en Chile neoliberal. La implementación del Convenio 169 de la OIT desde la perspectiva de dirigentes Mapuche Williche. Cultura-hombre-sociedad, 2018, vol. 29, no. 1, p. 108-130. 
ALFRED, T. Wasáse: indigenous pathways of action and freedom. Toronto: University of Toronto Press, 2009.

ALLAIN, M. Conflictos y protestas socio-ambientales en Chile: Reflexiones metodológicas y resultados. Revista de Sociología, vol. 34, no. 1, 2019, p. 81-101.

ÁLVAREZ, M. Las que han sabido aplicar el Convenio 169 (...) son empresas que van avanzando. El Austral. Temuco, Chile, 24 mayo, 2015. p. 14.

ARMONY, V. El multiculturalismo en las sociedades pluralistas. Centre MCD-UQÀM, 2001.

ASOCIACIÓN LEUFÜ WUENEYWUE. Asociación Leufü Wueneywue se manifiesta en defensa del río Wueneywue. El Ciudadano [en línea]. [Consulta: 29 diciembre 2020]. Disponible en: https:// www.elciudadano.com/chile/asociacion-leufu-wueneywue-se-manifiesta-en-defensa-del-rio-wueneywue/08/17/, 2018.

AYLWIN, J. Políticas Publicas y Pueblos Indígenas: El Caso De La Política De Tierras Del Estado Chileno Y El Pueblo Mapuche. University of Texas, Center for Latin American Social Policy (CLASPO), Unites States of America, 2002.

AYLWIN, J. La Política del «Nuevo Trato» Antecedentes, Alcances y Limitaciones. En: J. AYLWIN y N. YAÑEZ (eds.), El Gobierno de Lagos, los Pueblos Indígenas y el Nuevo Trato". Las Paradojas de la Democracia Chilena. Santiago de Chile: Lom Ediciones, 2005, p. 29-58.

BELLO, Á. El Programa Orígenes y la política pública del gobierno de Lagos hacia los pueblos indígenas. En: N. YAÑEZ y J. AYLWIN (eds.), El Gobierno de Lagos, los pueblos indígenas y el «nuevo trato». Las paradojas de la democracia chilena. Santiago: Lom Ediciones, 2007, p. 193-220.

BIBLIOTECA DEL CONGREO NACIONAL DE CHILE. Historia de la Ley № 19.940 Regula sistemas de transporte de Energía Eléctrica, establece un nuevo régimen de tarifas para sistemas eléctricos medianos e introduce las adecuaciones que indica a la ley general de servicios eléctricos: Disponible en: https://www.bcn.cl/historiadelaley/nc/historia-de-la-ley/5720/, 2004.

BOCCARA, G.. Le gouvernement des "Autres ". Sur le multiculturalisme néolibéral en Amérique Latine, Abstract. Actuel Marx, no. 50, 2011, p. 191-206.

BOCCARA, G. y BOLADOS, P. ¿Qué es el multiculturalismo? La nueva cuestión étnica en el Chile neoliberal. Revista de Indias, vol. 70, no. 250, 2010, p. 651-690.

CARMONA CALDERA, C.. La aplicación del derecho a consulta del Convenio 169 de la OIT en Chile. Hacia una definición de su contenido sustantivo: afectación e instituciones representativas. Tesis de Maestría. UNSAM, 2013

COLLINAO, E. Enel Green Power ofrece migajas a las comunidades de Panguipulli [en línea]. Lian Producciones. [Consulta: 28 diciembre 2020]. Disponible en: https://www.youtube.com/watch?$\mathrm{v}=\mathrm{Q} 6 \mathrm{RLh} 4 \mathrm{FAjDQ} \& \mathrm{t}=2 \mathrm{~s}, 2014$. 
COMISIÓN DE EVALUACIÓN REGIÓN DE LOS RíOS. Resolución Exenta № 008 del 02 marzo del 2017 [en línea]. 2017. S.I.: s.n. Disponible en: https://seia.sea.gob.cl/archivos/2017/03/08/Res._ Ex._008_2017_Resuelve_Inval._Florin.pdf, 2014.

COMISIÓN NACIONAL DE ENERGÍA. Reporte Capacidad Instalada Generación, Diciembre [en línea]. 2020. S.I.: s.n. Disponible en: https://www.cne.cl/wp-content/uploads/2020/12/Capacidad_ Instalada_Generaci\%C3\%B3n.xlsx, 2020.

COMISIONADO PRESIDENCIAL PARA ASUNTOS INDÍGENAS. Informa de la Comisión Verdad Histórica y Nuevo Trato con los Pueblos Indígenas. [en línea]. Santiago de Chile: [Consulta: 26 abril 2019]. Disponible en: http://www.memoriachilena.gob.cl/602/articles-122901_recurso_2.pdf, 2008.

CONSEJO DE COMUNIDAdES PULLINQUE. Video Declaración Pública Pullinque: Recuperando los espacios y el agua usurpada por Enel. El Puelche [en línea]. [Consulta: 28 diciembre 2020]. Disponible en: https://www.elpuelche.cl/2017/01/20/video-declaracion-publica-pullinque-recuperando-los-espacios-y-el-agua-usurpada-por-enel/, 2017.

CONSEJO DE LA CORPORACIÓN DE FOMENTO DE LA PRODUCCIÓN. Fomento de la Producción de Energía Eléctrica. Editorial Nascimiento, 1939.

COULTHARD, G.S. Peau rouge, masques blancs: contre la politique coloniale de la reconnaissance. Montreal: Lux Éditeur. Humanités, 2018.

CUADRA MONTOYA, X.A., Pluralisme radical et décolonisation dans les mobilisations autochtones face à l'industrie extractive : analyse de deux cas au Chili et au Québec. Memoria de maestría. Département de science politique. Université du Québec à Montréal, 2014.

CUADRA MONTOYA, X.A. La revendication autochtone contre le projet hydro-électrique Neltume au Chili: Un regard décolonial sur les antagonismes sociaux. Cahiers du CIÉRA, vol. 13, 2016, p. 60-78.

CUSICANQUI, S.R., 2016. Etnicidad Estratégica, Nación y (Neo)Colonialismo en América Latina. Alternativa. Revista de Estudios Rurales, no. 5, 2016, p. 65-87.

DELAMAZA, G., MAILLET, A. y MARTíNEZ NEIRA, C. Socio-Territorial Conflicts in Chile: Configuration and Politicization (2005-2014). ERLACS, vol. 0, no. 104, 2017, pp. 23.

DIRECTOR EJECUTIVO SERVICIO DE EVALUACIÓN AMBIENTAL. Instructivo sobre Implementación del Proceso de Consulta de Pueblos Indígenas en conformidad con el Convenio 169 de la OIT en el Sistema de Evaluación de Impacto Ambiental. [en línea]. Santiago de Chile: Servicio de Evaluación Ambiental. [Consulta: 30 abril 2019]. Disponible en: https://www.sea.gob.cl/sites/default/ files/migration_files/instructivos/of._ord._ndeg_161116.pdf, 2016.

DOYTCHEVA, M.. Le multiculturalisme. Paris: La Découverte, 2011. 
ENDESA. Pilmaiquén, Central Hidroeléctrica. Empresa Editora Zig-Zag, 1944.

ENEL. Pilmaiquén: Lugar de encuentro con la cultura mapuche. Enel/Historias [en línea]. [Consulta: 6 noviembre 2020]. Disponible en: https://www.enel.cl/content/enel-cl/es/megamenu/historias/archive/2019/02/Pilmaiquen-Lugar-de-encuentro-con-la-cultura-mapuche.html, 2019.

GARCÍA PETER, S.. El multiculturalismo como modelo de gobernanza en Chile: Estado, Academia y Brokers. Universitas Humanística, no. 82, 2016.

GIMINIANI, P.D. The becoming of ancestral land: Place and property in Mapuche land claims. American Ethnologist, vol. 42, no. 3, 2015, p. 490-503.

GLASER, B.G. y STRAUSS, A.L.. The discovery of grounded theory strategies for qualitative research. Chicago: Chicago Aldine Pub. Collections : Observations, 1967.

GUDYNAS, E. Extracciones, extractivismos y extrahecciones. Un marco conceptual sobre apropiación de recursos naturales. Observatorio del Desarrollo, no. 18, 2013, p. 1-18.

GOBIERNO DE CHILE. Re-Conocer. Pacto Social por la Multiculturalidad. [en línea]. Santiago de Chile: Disponible en: http://www.intendencialaaraucania.gov.cl/filesapp/Pacto\%20social.pdf, 2008.

GONZÁLEZ, V. y MOLINA, M. Tras 7 años de conversaciones: comunidad Mapu Pilmaiquén inaugura Parque La Isla - Salto La Olla. BioBioChile [en línea]. [Consulta: 6 noviembre 2020]. Disponible en: https://www.biobiochile.cl/noticias/nacional/region-de-los-lagos/2019/02/21/tras-7-anos-de-conversaciones-comunidad-mapu-pilmaiquen-inaugura-parque-la-isla-salto-la-olla.shtml, 2019.

GUERRA SCHLEEF, F.A. Los Tribunales Ambientales en la implementación de los derechos indígenas durante la evaluación ambiental de proyectos de inversión en Chile. Revista Justicia Ambiental, no. 9, 2017, p. 19-38.

HALE, C. y MILLAMÁN, R. Cultural Agency and Political Struggle in the Era of the Indio permitido. En: D. SOMMER (ed.), Cultural Agency In The Americas. Durham: Duke University Press, 2005. p. 281-304.

HALE, C.R . More Than an Indian. S.I.: Asociación para el Avance de las Ciencias Sociales en Guatemala, 2006.

HERNANDO ARRESSE, M. La vida social de los ríos y los conflictos hidroeléctricos en el Wallmapu. En: VILA BENITES, G. y Cristóbal BONELLI C. (editores). Contacorriente. Agua y Conflicto en América Latina. Quito: Abya Ayala, 2017, p. 135-155.

$\mathrm{HÖHL}, J$. Pueblos indígenas, recursos y gobernanza. Un análisis de la consulta indígena como parte de la Evaluación de Impacto Ambiental del proyecto hidroeléctrico Añihuerraqui, Región de la Araucanía, Chile. Investigaciones Geográficas, no. 59, 2020, p. 28-40. 
IBARRA ELIESSETCH, M.I. y RIQUELME MAULÉN, W. Sentipensar mapuche con las aguas del Huenehue: Hacia una ecología política y una antropología por demanda. Polis. Revista Latinoamericana, no. 54., 2019.

INSTITUTO NACIONAL DE DERECHOS HUMANOS. Mapa de Conflictos Socioambientales en Chile. [en línea]. Santiago de Chile: INDH. [Consulta: 9 abril 2018]. Disponible en: http://mapaconflictos. indh.cl/assets/pdf/libro-web-descargable.pdf, 2015.

KELLY, S., Articulating Indigenous Rights Amidst Territorial Fragmentation: Small Hydropower Conflicts in the Puelwillimapu, Southern Chile. Tucson, Arizona: University of Arizona. Tesis para optar al grado de doctorado, 2018.

KELLY, S. Megawatts mask impacts: Small hydropower and knowledge politics in the Puelwillimapu, Southern Chile. Energy Research \& Social Science, vol. 54, 2019, p. 224-235.

KYMLICKA, W., 1995. Multicultural Citizenship. A liberal Theory of Minority Rights. Oxford: Oxford University Press, 1995.

KYMLICKA, W. Neoliberal Multiculturalism? En: P.A. HALL y M. LAMONT (eds.), Social Resilience in the Neoliberal Era. Cambridge University Press. Cambridge, 2013, p. 99-126.

KYMLICKA, W. The Essentialist Critique of Multiculturalism: Theories, Policies, Ethos. En: V. UBEROI y T. MODOOD (eds.), Multiculturalism Rethought Interpretations, Dilemmas and New Directions. Edinburgh: Edinburg University Press, 2015, p. 209-249.

LACLAU, E. y MOUFFE, C. Hegemonía y estrategia socialista. Hacia una radicalización de la democracia. Buenos Aires: Fondo de Cultura Económica, 2011.

MADARIAGA, A. y GLADINA, E. La transformación de la política energética como cambio de paradigma. En: F. GONZÁLEZ Y A. MADARIAGA (EDS.) y A. MADARIAGA (eds.), La constitución política, social y moral de la economía chilena. Santiago de Chile: Ril Editores/Universidad Central de Chile, 2018, p. 379-408.

MADRID MESCHI, A. Recurso de Invalidación de la Comunidad Benancio Huenchupan ante la Comiisón de Evaluación Ambiental Región de la Araucanía [en línea]. 2019. S.I.: s.n. Disponible en: https://seia.sea.gob.cl/archivos/2019/11/27/Invalidacion_Cl_Benancio_Huenchupan.pdf, 2019.

MAILLET, A. Variedades de neoliberalismo. Innovación conceptual para el análisis del rol del Estado en los mercados. Revista de Estudios Políticos, no. 169, 2015, p. 109-136

MAILLET, A. y ROZAS BUGUEÑO, J. Hibridación de las políticas neoliberales. El caso de la reforma a la política eléctrica en Chile (2014-2016). Gestión y Política Pública, vol. 28, no. 1, 2019, p. 207-235.

MAPUEXPRESS. Comisión Cámara sesionara en la Araucanía y citará a Ministros de Energía y Medio Ambiente por conflicto hidroeléctrico. Mapuexpress [en línea]. [Consulta: 13 marzo 2020]. 
Disponible en: https://www.mapuexpress.org/2016/01/21/comision-camara-sesionara-en-la-araucania-y-citara-a-ministros-de-energia-y-medio-ambiente-por-conflicto-hidroelectrico/, 2019.

MARIMÁN, P. Los Mapuche antes de la conquista militar chileno-argentina. En: P. MARIMÁN, S. CANIUQUEO, J. MILLALÉN y R. LEVIL (eds.), i... Escucha, Winka...! Cuatro Ensayos de Historia Nacional Mapuche y Un Epílogo Sobre el Futuro. Santiago: Lom Ediciones, 2006, p. 53-127.

MEZA-LOPEHANDÍA GLAESSER, M. La Jurisprudencia Del Multiculturalismo En Chile: La Consulta Previa Indígena Ante Tribunales. Revista de Ciencias Sociales [en línea], no. 69. 2016, p. 13-52.

MILLAMÁN MANRÍQUEZ, S.E. Consulta Indígena, Pueblo Mapuche, Territorios Y Evaluación Medioambiental. [en línea]. Documento de Trabajo. Santiago: Instituto de Investigación en Ciencias Sociales Universidad Diego Portales. Serie Laboratorio Constitucional, 2019, 52.

MINISTERIO DE ENERGÍA. Estrategia Nacional de Energía 2012-2030. Energía para el Futuro. [en línea]. Santiago, Chile: [Consulta: 9 abril 2019]. Disponible en: https://mma.gob.cl/wp-content/ uploads/2014/10/3_Estrategia-Nacional-de-Energia-2012-2030_Energia-para-el-Futuro.pdf, 2012.

MINISTERIO DE ENERGÍA. Energía 2050. Política Energética de Chile. [en línea]. Santiago de Chile: Gobierno de Chile. Disponible en: http://www.energia.gob.cl/sites/default/files/energia_2050_-politica_energetica_de_chile.pdf, 2015.

MINISTERIO DE ENERGÍA. Compromiso de Diálogo. Estándar de Participación para el Desarrollo de Proyectos de Energía [en línea]. Santiago de Chile: Gobierno de Chile. Disponible en: http://www. minenergia.cl/archivos_bajar/2016/Documentos/CompromisoDialogoEstandarParticipacion.pdf, 2016.

MINISTERIO DE ENERGÍA. Capítulo Indígena de la Política Energética 2050 [en línea]. Santiago de Chile: Gobierno de Chile. Disponible en: http://www.energia.gob.cl/sites/default/files/capitulo-de-pertinencia-indigena-de-la-politica-energetica-nacional.pdf, 2017.

MITCHELL, K.. Crossing the neoliberal line: Pacific Rim migration and the metropolis [en línea]. Philadelphia: Temple University Press, 2004.

MOUFFE, C. En torno a lo político. Buenos Aires: Fondo de Cultura Económica, 2007.

NAPADENSKY PASTENE, A. Paisajes rurales y produccion energética. Luces y sombras de una transformación en proceso. Revista de Desarrollo Rural y Cooperativismo Agrario, vol. 11, 2007, p. 123-132.

NORVAL, A.J. Social Ambiguity and the Crisis of Apartheid. En: E. LACLAU (ed.), The Making of Political Identities. London-New York: Verso, 1994, p. 115-137.

NUÑEZ, D. Impulso a la Inversión. En: M. PACHECO M. (ed.), Revolución Energética en Chile. Santiago de Chile: Ediciones Universidad Diego Portales, 2018, p. 481-506. 
OPASO, C. Biobío Rebelde: De Ralquil a Ralco. Ceibo, 2012.

ORELLANA, M.A. Indigenous Peoples, Energy and Environmental Justice: The Pangue/Ralco Hydroelectric Project in Chile's Alto BíoBío. Journal of Energy \& Natural Resources Law, vol. 23, no. 4, 2005, p. 511-528.

OSORNO TV. Nota Comunidad Mapu Pilmaiquén Inaugura Parque La Isla - Salto La Olla [en línea]. Pilmaiquén: [Consulta: 6 noviembre 2020]. Disponible en: https://www.youtube.com/watch?v=DmEdU3sITLA, 2019.

PERAlTA, C. La Asimetría del Poder. Conflicto Etno-ambiental en el Marco de la Consulta Indígena: Caso Central Hidroeléctrica Añihuerraqui, Curarrehue. Tesis de Maestría. Santiago, Chile: Universidad Diego Portales, 2015.

POSTERO, N. Now We Are Citizens, Indigenous Politics in Postmulticultural Bolivia. Stanford University Press. Stanford, CA, 2007.

PRIETO, M. y BAUER, C. Hydroelectric power generation in Chile: an institutional critique of the neutrality of market mechanisms. Water International, vol. 37, no. 2, 2012, p. 131-146.

PROGRAMA DE DERECHOS INDÍGENAS. Los Derechos de Los Pueblos Indígenas en Chile. Temuco-Santiago: Instituto de Derechos Indígenas de la Universidad de La Frontera - Lom, 2003.

RICHARDS, P. Race and the Chilean Miracle: Neoliberalism, Democracy, and Indigenous Rights. S.I.: University of Pittsburgh Press, 2013.

RUDNICK, H. y PALMA, R. Contexto Nacional e Internacional de marzo de 2014. En: M. PACHECO M. (ed.), Revolución energética en Chile. Santiago, Chile: Ediciones Universidad Diego Portales, 2018, p. 19-40.

SERVICIO DE EVALUACIÓN AMBIENTAL. Proyectos con Proceso de Consulta a Pueblos Indígenas. Sistema de Evaluación de Impacto Ambiental e-seia [en línea]. [Consulta: 31 enero 2021]. Disponible en: https://seia.sea.gob.cl/pci/proyectos_en_pci.php, 2020

SIEDER, R. Multiculturalism in Latin America - Indigenous Rights, Diversity and Democracy. London: Palgrave Macmillan UK, 2002.

SILVA NERIZ, H. La Jurisprudencia De Los Tribunales De Justicia En Chile En Materia De Consulta: Un Largo Y Complejo Camino. En: OBSERVATORIO CIUDADANO (ed.), El Derecho A La Consulta De Los Pueblos Indígenas: Análisis Del Derecho Nacional, Internacional y Comparado. Temuco, 2014, p. 47-58.

SONEIRA, A.J. La "Teoría fundamentada en los datos» (Grounded Theory) de Glaser y Strauss. En: I. VASILACHIS DE GIALDINO (ed.), Estrategias de investigación cualitativa. Barcelona: Gedisa, 2006, p. 153-174. 
SOTO, H. Los 18 proyectos hidroeléctricos que apuntan a la matriz regional. El Austral. Temuco, Chile, 17 diciembre, 2015. p. 2-3.

STRAUSS, A. y CORBIN, J. Les fondement de la recherché qualitative. Techniques et procédures de développement de la théorie enracinée. . Fribourg: Academic Press Fribourg., 2004.

SVAMPA, M. Consenso de los commodities, giro ecoterritorial y pensamiento crítico en América Latina. OSAL-Observatorio Social de América Latina, no. 32 (Noviembre), 2012, p. 15-38.

ROSEBERRY, W. Hegemonía y lenguaje contencioso. En: G. JOSEPH y D. NUGENT (eds.), Aspectos cotidianos de la formación del Estado. México D.F.: Ediciones Era, 2002, p. 213-226.

TAYLOR, C. Multiculturalism. Examining the Politics of Recognition. S.I.: Princeton University Press, 1994.

THOMASSEN, L. British Multiculturalism and the Politics of Representation. Edinburgh: Edinburgh University Press, 2017.

TIRONI, M. y PIRCOVIĆ, T. Conflictos energéticos en Chile. Estudio base para el periodo 20002015. [en línea]. Documento de Trabajo NUMIES, №2. NUMIES, 2017.

TOKMAN, M. Política Energética: Nuevos Lineamientos. Transformando la Crisis Energética en una Oportunidad Política. Santiago de Chile [en línea]. 2008. S.I.: Comisión Nacional de Energía. [Consulta: 5 abril 2019]. Disponible en: https://www.cne.cl/archivos_bajar/Politica_Energetica_Nuevos_ Lineamientos_08.pdf, 2008.

VAN COTT, D.L. The Friendly Liquidation of the Past: The Politics of Diversity in Latin America. Pittsburgh: University of Pittsburgh Press, 2000.

VÁSQUEZ, V., VALDEBENITO, G. y OGALDE, M. Paisajes culturales: la instalación de la central hidroeléctrica Llollelhue, La Turbina, en la ciudad de La Unión. Revista de Urbanismo, no. 38,2018, p. 1-16.

YAÑEZ, C. El arranque del sector eléctrico chileno. Un enfoque desde las empresas de generación, 1897-1931. En: D. BARRÍA TRAVERSO y M. LLORCA-JAÑA (eds.), Empresas y empresarios en la historia de Chile: 1810-1930. Santiago: Editorial Universitaria, 2017a, p. 175-193.

YAÑEZ, C.. La intervención del Estado en el sector eléctrico chileno. Los inicios de la empresa pública monopólica. En: M. LLORCA-JAÑA y D. BARRÍA TRAVERSO (eds.), Empresas y empresarios en la historia de Chile: 1930-2015. Santiago: Editorial Universitaria, 2017b, p. 109-132.

ŽIŽEK, S. Multiculturalismo o la lógica cultural del capitalismo multinacional. En: F. JAMESON y S. ŽIŽEK (eds.), Estudios Culturales, 1998, p. 137-188. 
